\title{
Phase Singularities in a Cardiac Patch Model With a Non-conductive Fibrotic Area During Atrial Fibrillation
}

\author{
Tiago P Almeida ${ }^{1,2,3 *}$, Mark Nothstein ${ }^{4 *}, \mathrm{Xin} \mathrm{Li}^{1,3}$, Michela Masè ${ }^{5}$, Flavia Ravelli ${ }^{6}$, Diogo C \\ Soriano $^{7}$, Arthur S Bezerra ${ }^{2}$, Fernando S Schlindwein ${ }^{1,3}$, Takashi Yoneyama ${ }^{2}$, Olaf Dössel ${ }^{4}$, \\ $\mathrm{G}$ André $\mathrm{Ng}^{1,3}$, Axel Loewe ${ }^{4}$ \\ ${ }^{1}$ University of Leicester, UK; ${ }^{2}$ Instituto Tecnológico de Aeronáutica, Brazil; ${ }^{3}$ NIHR Leicester \\ Cardiovascular Biomedical Research Centre, UK; ${ }^{4}$ Karlsruhe Institute of Technology, KIT, Germany; \\ ${ }^{5}$ Eurac Research, Italy; ${ }^{6}$ Università degli Studi di Trento, Italy; ${ }^{7}$ Federal University of $\mathrm{ABC}$, Brazil
}

\begin{abstract}
In-silico cardiac patches with a non-conductive fibrotic areas were used to investigate the occurrence and characteristics of rotors described by phase singularities $(P S s)$. A rotor was generated in a virtual tissue patch represented by a Courtemanche model adapted to mimic AF conditions. Non-conductive fibrotic elements were incorporated around the center of the patch (densities of $20 \%, 30 \%$, and 40\%). Electrograms (EGMs) were calculated and their phase was determined using Hilbert transform. PSs were detected and PS density maps (PSDMs) were generated. Six attributes were used to investigate bipolar EGMs obtained from the original signals: sample entropy (SampEn), determinism (DET), peak-to-peak (PP), wave similarity (WS), organization index (OI), and cycle length $(C L) .77 .1 \%$ of the PSs occurred inside the fibrotic region - with a higher density in the border zone - for $20 \%$ density; $99.8 \%$ for 30\%; 99.7\% for $40 \%$. SampEn was significantly higher in regions with $P S D M \geq 5$, while DET, $P P$, and WS were significantly lower $(P<0.0001)$. PSs tended to meander outside the fibrotic area for lower fibrotic density. Bipolar EGM attributes offer a complementary way for rotor detection in the presence of non-conductive fibrosis.
\end{abstract}

\section{Introduction}

Multiple mechanisms are believed to participate in the perpetuation of atrial fibrillation (AF) - the most common heart rhythm disorder seen in clinical practice - which poses challenges for the correct identification of targets for AF ablation therapy [1]. Recent works have suggested that areas with reentrant activity (i.e., rotors) represent important sites for ablation [2], and recent investigations have linked the occurrence of such rotors to cardiac regions with fibrotic tissue [3].

Fibrosis represents a complex structure comprised of different factors - such as collagen deposition, inflammatory cytokines, proteins, among others - that alter the electrophysiology of the cardiac tissue. For instance, heterogeneities induced by the presence of fibrosis alter conduction patterns in the cardiac tissue, creating regions of slow and anisotropic conduction [4]. Such delayed activation might favor the occurrence of re-entrant activity, which in turn participates in the perpetuation of the arrhythmia [5]. The relationship between rotors and fibrosis - as well as rotor detection related to fibrosis remains controversial [4]. Additionally, methods for automated rotor detection during cardiac electrophysiologic studies rely mostly on unipolar measurements, whereas the majority of clinical centers perform AF ablation guided by attributes extracted from bipolar electrograms (EGMs) [2]. Computational modeling may represent a valuable tool to investigate these aspects, in which the ground truth for remodeled cardiac tissue is known [6].

In the present work, in-silico cardiac patches with nonconductive fibrotic area were used to investigate the occurrence and characteristics of rotors described by phase singularities (PSs). Bipolar EGM attributes were used to characterize the influence of PSs in the cardiac tissue.

\section{Methods}

\subsection{Non-conductive fibrotic tissue in a virtual cardiac patch}

A stable rotational source was generated by cross field stimulus protocol [7] in an isotropic virtual tissue patch measuring $30 \mathrm{~mm} \times 30 \mathrm{~mm} \times 2 \mathrm{~mm}$, with a spatial resolution of $100 \mu \mathrm{m}(0.1 \mathrm{~mm} \times 0.1 \mathrm{~mm} \times 0.1 \mathrm{~mm})$, as previously described in [4].

Membrane kinetics were represented by the Courtemanche et al. model [8], adapted to mimic persAF conditions [9]. The conductivity of the non-fibrotic region 


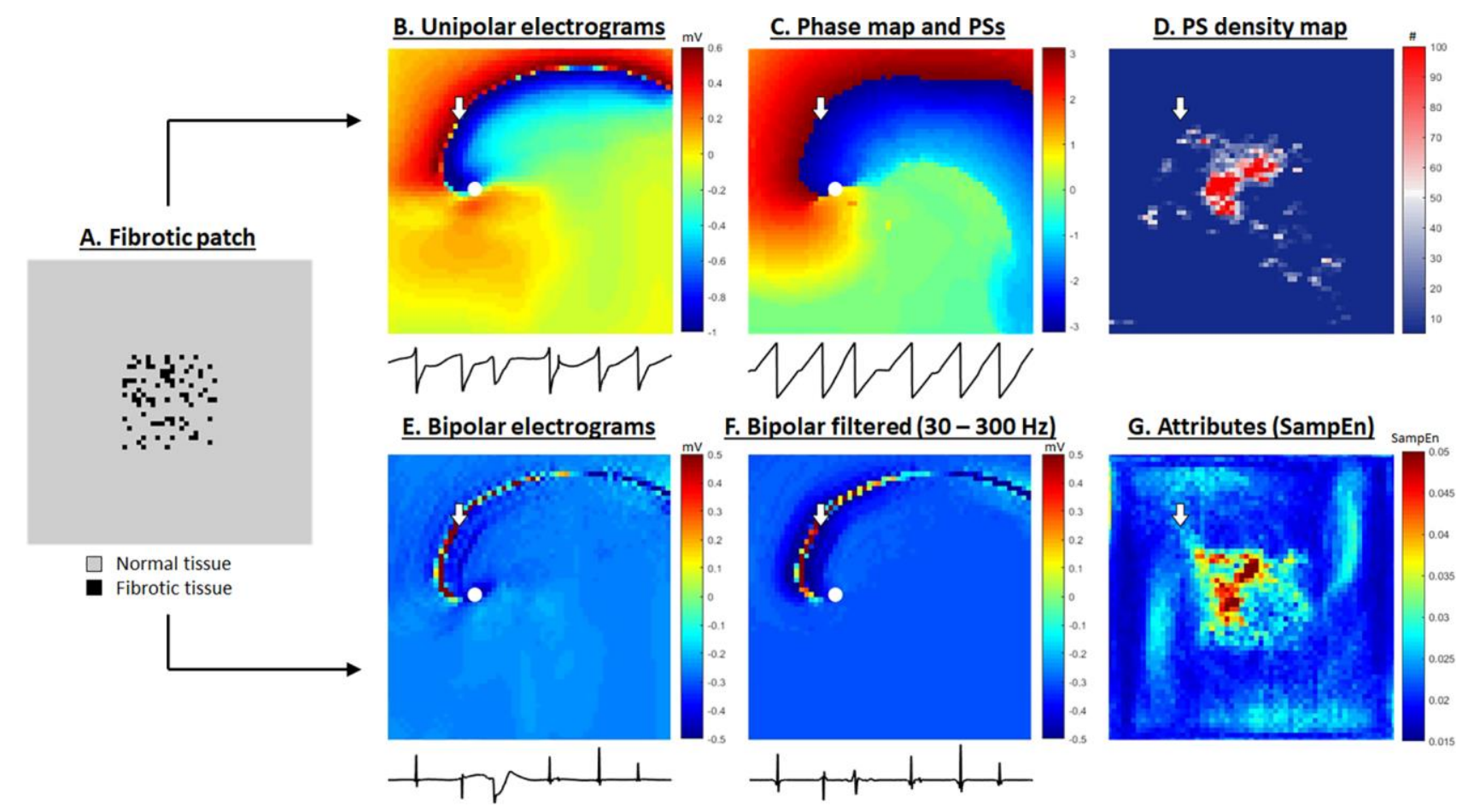

Figure 1. Illustration of a single time frame of the processing steps for analyzing the relationship between non-conductive fibrosis and PS occurrence using bipolar EGMs attributes for $20 \%$ of fibrotic density. A. The original patch (non-conductive fibrotic tissue highlighted in black). B. A time frame showing the unipolar voltage during an induced fibrillatory event. The PS at the time frame is highlighted with the white sphere and the location of the illustrated EGM is highlighted with the white arrow. C. The corresponding phase map. D. The PS density map for the entire $8 \mathrm{~s}$ episode. E. and F. The resulting bipolar voltage maps of the same time frame with the original and filtered bipolar EGMs, respectively. G. One of the attributes extracted from the filtered bipolar EGMs (SampEn).

was adapted to yield a plane wave conduction velocity of $0.22 \mathrm{~m} / \mathrm{s}$. Regional fibrosis was incorporated into the model within an area of $10 \mathrm{~mm} \times 10 \mathrm{~mm} \times 2 \mathrm{~mm}$ at the center of the patch and was modelled by setting the conductivity for each fibrotic element to zero (Figure 1A). The volume fraction of these fibrotic elements was set to $20 \%, 30 \%$ and $40 \%$ (higher densities induce full conduction block). Uniform distribution of transmural 100 $\mu \mathrm{m} \times 100 \mu \mathrm{m}$ non-conductive elements within the fibrotic area was considered. The size of non-conductive elements was adapted to approximate histological observations on collagen septa in aged human atria [10]. Excitation propagation was simulated using the cardiac electrophysiology solver acCELLerate [11]. Eight seconds of extracellular potentials were sampled at $1 \mathrm{kHz}$ and calculated in direct contact with the endocardial surface, with an inter-electrode distance of $0.5 \mathrm{~mm}$, resulting in a 2D mesh with 60 x 60 unipolar EGMs (Figure 1B).

\subsection{Signal processing}

The phase of the unipolar EGMs was determined using sinusoidal wavelet reconstruction followed by Hilbert transform [12]. PSs were detected using the topological charge method (Figure 1C) [13] and PS density maps
(PSDMs) were generated (Figure 1D) from 8 seconds of electrogram data.

Bipolar EGMs were obtained from the unipolar signals (Figure 1E) and filtered following the clinical recommendations (30-300 Hz, Figure 1F). Attributes were extracted from the filtered bipolar EGMs (Figure 1G), as described in the following section.

\subsection{Attributes for bipolar EGMs}

A set of attributes, previously proposed as AF markers, were selected to quantitatively characterize the filtered bipolar EGMs collected in regions with $v s$. without PSs:

1) Sample entropy (SampEn) provides a direct estimation of signal amplitude distribution - signal complexity [14].

2) Determinism (DET). Based on recurrence quantification analysis, it characterizes phase transitions and underlying nonlinear phenomena. It was shown to be effective in discriminating turbulent behavior in AF EGMs [15].

3) Organization index (OI) is a measure of signal spectrum dispersion, bounded between 0 and 1 , with smaller values indicating more fractionated EGMs [14].

4) Wave similarity (WS) is a measure of the repetitiveness of local activation wave morphology in AEGs, which is reflective of the organization of the underlying conduction 
patterns [16].

5) Peak-to-peak (PP) amplitude voltage is extracted from local activation waveforms and quantifies the AEG voltage amplitude. It might help to identify low voltage zones, which are believed to correlate with the fibrosis presence [14].

6) Cycle length (CL) represents the rate of activation of the underlying atrial tissue estimated in the time-domain [19]. 7) Interval confidence level (ICL), average complex interval (ACI), and shortest complex interval (SCI) are the clinical attributes calculated by the CARTO mapping system to measure AEG fractionation [14].

\subsection{Statistical analysis}

All continuous non-normally distributed variables are expressed as median and interquartile interval. Nonparametric unpaired multiple data were analyzed using the Kruskal-Wallis test with Dunn's correction. Receiver operating characteristic (ROC) curves were created considering the density of PSs (PSDM $\geq 5$ ) as the reference for classification and the EGM attributes as predictors. The area under the ROC (AUROC) curves were calculated to assess the quality of the discriminators. P-values $<0.05$ were considered statistically significant.

\section{Results}

PSs were more concentrated inside the fibrotic region at higher fibrosis densities: $77.1 \%$ of the PSs occurred inside the fibrotic region in the patch with $20 \%$ fibrotic density (Figure 1D), 99.8\% in the patch with $30 \%$ density, and $99.7 \%$ in the one with $40 \%$ density. In all cases PSs occurred more often at the border zone of fibrotic regions. The PSs tended to meander less at increasing fibrotic density: PS trajectories covered $64 \%$ of the fibrotic region in the patch with $20 \%$ fibrotic density, $35 \%$ for $30 \%$ density, and $28 \%$ for $40 \%$ density.

The presence of PSs induced significant changes in EGM amplitude, dynamics, and morphology, as highlighted by the EGM attributes in Figure 2. SampEn was significantly higher for EGMs collected in regions with $\mathrm{PSDM} \geq 5$ (Figure $1 \mathrm{H}$ ), while DET, PP, and WS were significantly lower $(\mathrm{P}<0.0001)$. OI was significantly lower in PS regions for $30 \%$ and $40 \%$ densities $(\mathrm{P}<0.0001)$, while there were no significant differences between regions with and without PSs in terms of CL. The clinical attributes calculated by CARTO showed significant differences $(\mathrm{P}<0.0001)$ between regions with and without PSs for $20 \%$ and $30 \%$ fibrotic densities.

The AUROC curves highlighted the quality of each attribute as discriminator for classifying regions with PSDM $\geq 5$ (Table 1). SampEn, DET, WS, and PP were accurate discriminators, while $\mathrm{OI}, \mathrm{CL}$, and the clinical attributes (ICL, ACI and SCI) were not as effective.
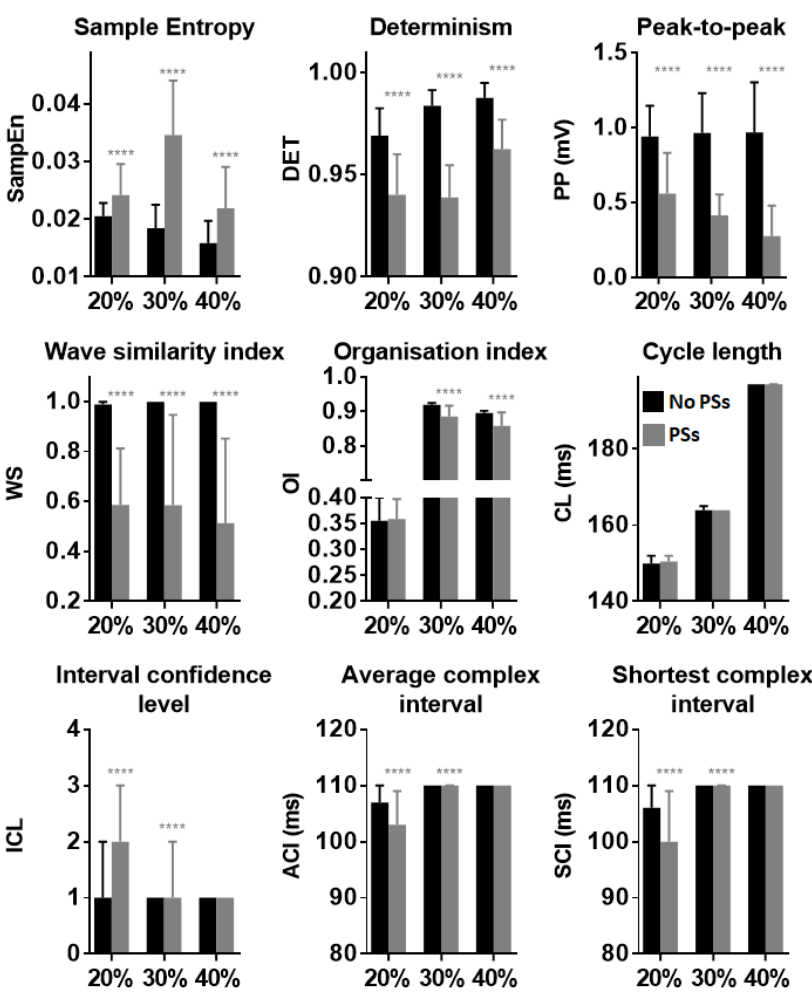

Figure 2. EGM attributes calculated in regions with (grey) and without (black) PSs in patches with different fibrotic densities $(20 \%, 30 \%$, and $40 \%)$. **** $\mathrm{p}<0.0001$.

Table 1. The AUROC curve for the EGM attributes when discriminating regions with $v s$. without PSs in different fibrotic density. The highest AUROC curves for each density are highlighted.

\begin{tabular}{cccc}
\hline \hline \multirow{2}{*}{ Attributes } & \multicolumn{3}{c}{ Fibrotic density } \\
\cline { 2 - 4 } & $20 \%$ & $30 \%$ & $40 \%$ \\
\hline \hline SampEn & 0.80 & $\mathbf{0 . 9 2}$ & 0.72 \\
DET & 0.71 & 0.87 & 0.81 \\
OI & 0.55 & 0.75 & 0.77 \\
WS & $\mathbf{0 . 8 1}$ & 0.89 & $\mathbf{0 . 9 4}$ \\
CL & 0.52 & 0.62 & 0.67 \\
PP & 0.71 & 0.82 & 0.89 \\
\hline ICL & 0.64 & 0.64 & 0.53 \\
ACI & 0.69 & 0.73 & 0.56 \\
SCI & 0.69 & 0.73 & 0.56 \\
\hline \hline
\end{tabular}

\section{Discussion and conclusions}

Our results suggest that attributes extracted from filtered bipolar EGMs - broadly used to characterize the atrial substrate in electrophysiologic studies - are effective in detecting regions with rotors in the presence of nonconductive fibrosis. Additionally, our results show that complementary attributes - such as SampEn and WS should be preferred to characterize AF rotors over clinical 
attributes calculated by electroanatomic commercial systems - such as CARTO. More importantly, while CL is frequently used as a measure for AF complexity, it showed poor performance in characterizing regions with rotors. These results might partially explain the methodological heterogeneities in rotor-guided ablation and the subsequent inconsistencies in ablation outcomes.

Recent works have shown the potential benefits of multiparametric analysis for a more thorough characterization of the atrial substrate using multiple EGM attributes [14]. The combination of the proposed attributes - but not limited to them - in a classification model might improve target identification for AF ablation using filtered bipolar EGMs.

\section{Acknowledgments}

TPA, DCS and ASB received research grants from FAPESP (Brazil, 2017/00319-8, 2018/02251-4; 2019/09512-0; 2019/05192-1). TPA received support from the BHF (UK, PG/18/33/33780 and the BHF Research Accelerator for ECR). XL received research grants from MRC (UK, DPFS MR/S037306/1). FR received research grant from Fondazione Cassa di Risparmio di Trento e Rovereto (2016.0273). GAN received research fellowship from St. Jude Medical and speaker fees and honoraria from Biosense Webster.

\section{References}

[1] H. Calkins, G. Hindricks, R. Cappato, Y. H. Kim, E. B. Saad, L. Aguinaga, et al., "2017 HRS/EHRA/ECAS/APHRS/SOLAECE expert consensus statement on catheter and surgical ablation of atrial fibrillation," Heart Rhythm, vol. 14, pp. e275-e444, Oct 2017.

[2] S. M. Narayan, D. E. Krummen, K. Shivkumar, P. Clopton, W. J. Rappel, and J. M. Miller, "Treatment of atrial fibrillation by the ablation of localized sources: CONFIRM (Conventional Ablation for Atrial Fibrillation With or Without Focal Impulse and Rotor Modulation) trial," J Am Coll Cardiol, vol. 60, pp. 628-36, Aug 142012.

[3] A. Frontera, M. Takigawa, R. Martin, N. Thompson, G. Cheniti, G. Massoullié, et al., "Electrogram signature of specific activation patterns: Analysis of atrial tachycardias at high-density endocardial mapping," Heart Rhythm, 2017/08/08/ 2017

[4] A. Jadidi, M. Nothstein, J. Chen, H. Lehrmann, O. Dössel, J. Allgeier, et al., "Specific electrogram characteristics identify the extra-pulmonary vein arrhythmogenic sources of persistent atrial fibrillation - characterization of the arrhythmogenic electrogram patterns during atrial fibrillation and sinus rhythm," Scientific Reports, vol. 10, p. 9147, 2020/06/04 2020.

[5] S. Zahid, H. Cochet, P. M. Boyle, E. L. Schwarz, K. N. Whyte, E. J. Vigmond, et al., "Patient-derived models link re-entrant driver localization in atrial fibrillation to fibrosis spatial pattern," Cardiovasc Res, vol. 110, pp. 443-54, Jun 1
2016.

[6] O. Dössel, M. W. Krueger, F. M. Weber, M. Wilhelms, and G. Seemann, "Computational modeling of the human atrial anatomy and electrophysiology," Medical \& Biological Engineering \& Computing, vol. 50, pp. 773-799, August 01 2012.

[7] A. Loewe, M. Wilhelms, F. Fischer, E. P. Scholz, O. Dossel, and G. Seemann, "Arrhythmic potency of human ether-a-gogo-related gene mutations L532P and N588K in a computational model of human atrial myocytes," Europace, vol. 16, pp. 435-43, Mar 2014.

[8] M. Courtemanche, R. J. Ramirez, and S. Nattel, "Ionic mechanisms underlying human atrial action potential properties: insights from a mathematical model," Am J Physiol, vol. 275, pp. H301-21, Jul 1998.

[9] A. Loewe, M. Wilhelms, O. Dössel, and G. Seemann, "Influence of chronic atrial fibrillation induced remodeling in a computational electrophysiological model," Biomedizinische Technik, vol. 59, pp. S929-S932, 2014.

[10] M. S. Spach, J. F. Heidlage, P. C. Dolber, and R. C. Barr, "Mechanism of origin of conduction disturbances in aging human atrial bundles: experimental and model study," Heart Rhythm, vol. 4, pp. 175-85, Feb 2007.

[11] G. Seemann, F. B. Sachse, M. Karl, D. L. Weiss, V. Heuveline, and O. Dössel, "Framework for modular, flexible and efficient solving the cardiac bidomain equations using PETSc," in Progress in Industrial Mathematics at ECMI 2008. vol. 15, A. Fitt, J. Norbury, H. Ockendon, and E. Wilson, Eds., ed Berlin, Heidelberg: Springer, 2010, pp. 363-369.

[12] P. Kuklik, S. Zeemering, A. van Hunnik, B. Maesen, L. Pison, D. H. Lau, et al., "Identification of rotors during human atrial fibrillation using contact mapping and phase singularity detection: technical considerations," IEEE Trans Biomed Eng, vol. 64, pp. 310-318, Feb 2017.

[13] X. Li, T. P. Almeida, N. Dastagir, M. S. Guillem, J. Salinet, G. S. Chu, et al., "Standardizing single-frame phase singularity identification algorithms and parameters in phase mapping during human atrial fibrillation," Frontiers in Physiology, vol. 11, 2020-July-21 2020.

[14] T. P. Almeida, G. S. Chu, X. Li, N. Dastagir, J. H. Tuan, P. J. Stafford, et al., "Atrial electrogram fractionation distribution before and after pulmonary vein isolation in human persistent atrial fibrillation - $\mathrm{a}$ retrospective multivariate statistical analysis," Frontiers in Physiology, vol. 8, 2017-August-24 2017.

[15] T. P. Almeida, F. S. Schlindwein, J. L. Salinet, L. Xin, G. S. Chu, J. H. Tuan, et al., "Characterization of human persistent atrial fibrillation electrograms using recurrence quantification analysis," Chaos, vol. 28, pp. 085710-1085710-12, 2018.

[16] F. Ravelli and M. Masè, "Computational mapping in atrial fibrillation: how the integration of signal-derived maps may guide the localization of critical sources," EP Europace, vol. 16, pp. 714-723, 2014.

Address for correspondence: Tiago Paggi de Almeida University of Leicester,

Leicester, UK

tpda2@le.ac.uk
Mark Nothstein

Karlsruhe Institute of Technology

(KIT), Karlsruhe, Germany

publications@ibt.kit.edu 